\title{
Background and Foreground Knowledge in knowledge management
}

\author{
J. Zheng, M. Zhou, J. Mo, A. Tharumarajah \\ CSIRO Manufacturing Science and Technology, Australia \\ Email: Jeffrey.Zheng@cmst.csiro.au
}

Keywords Implicit, Explicit, Tacit, Knowledge in Knowledge Management, Foreground, background, Executable, Non-executable, Dynamic Model of knowledge

\begin{abstract}
In this paper we propose a new dynamic model for knowledge in knowledge management. To analyze problems and relevant restrictions, two well-accepted knowledge representation models are discussed. The new model, Executable Knowledge Model (EKM), can distinguish foreground and background knowledge and it defines a determinable boundary for executable and nonexecutable knowledge in knowledge management. This model provides strong executable and testable properties of knowledge in knowledge management to guide further knowledge management development.
\end{abstract}

\section{INTRODUCTION}

What is knowledge? This fundamental question has raised such deep debate since the commencement of human history, inspiring the greatest philosophers such as Confucian, Plato, Aristotle, Russell, who have dedicated their lives to answer this question. Knowledge itself is a very slippery concept with many different variations and definitions. The nature of knowledge or what it means to know something is an epistemological question. Do we simply acknowledge that we are in an ambiguous area and do the best we can? No, we must each make these choices in an informed rational mindset for manipulation. In this field, there is no unique correct answer, only theories and opinions. With the rapid technology revolution, many researchers and commercial companies are starting to explore new management systems to handle and manipulate knowledge in order to support business activities. Knowledge management system seeks to efficiently handle practical knowledge. Since many knowledge models are 
mainly based on human beings and their experiences, there are intrinsic problems to directly use human oriented knowledge representation models to guide knowledge engineering practices in design and implementation of Knowledge Management Systems (KMS).

Knowledge models are applied to both nature and human beings. It is necessary to have executable principles to distinguish feasible functions in knowledge management with clear theoretical boundaries between human and machine. In this paper, a new model is proposed to satisfy these requirements. Two models (Nonaka 1991 and Nickols 2000) in knowledge management are evaluated. A new model (Executable Knowledge Model, EKM) is then proposed and applied for identifying foreground and background knowledge among management systems.

\section{TWO MODELS OF KNOWLEDGE MANAGEMENT}

Knowledge is the internal state of an agent following the acquisition and processing of information. An agent can be a human being, storing and processing information in his mind, or an abstract machine including devices to store and process information. To categorize human knowledge, Polanyi distinguishes that human knowledge has two major components: tacit and explicit knowledge [1,2].

\subsection{The Tacit and Explicit Loop}

Following Polanyi's concepts, one of the well-accepted theories about knowledge is proposed by Nonaka [3,4]. According to Nonaka, tacit knowledge consists of personal relationships, practical experience, shared values and explicit knowledge consists of formal policies and procedures. In Nonaka's knowledge model, the creation and classification is part of the externalization process. After having created or acquired tacit knowledge, human will put their ideas on paper. Retrieval is part of the internalization process. To show the dynamics for knowledge in knowledge creation, Nonaka's model can be illustrated in Figure 2.1.

In this model, the socialization has been approached in the context of organization culture. Combination is largely studied by the data processing (processing of formalized information, or data), internalization has been covered in the framework of the organization learning, but the last dimension, externalization (tacit formalization), has not been well developed yet. In order to do that, we can use techniques implemented inside the groupware systems to capture information to identify elements. We can also use mechanisms implemented in tools for meeting that allow capturing part of formulization of these elements (externalization). For a system, the aim of 
which is to help to capitalize enterprise knowledge and to create new knowledge (strategic resource), we must take into account not only the two static knowledge dimensions, but also the four dynamic knowledge interactions.

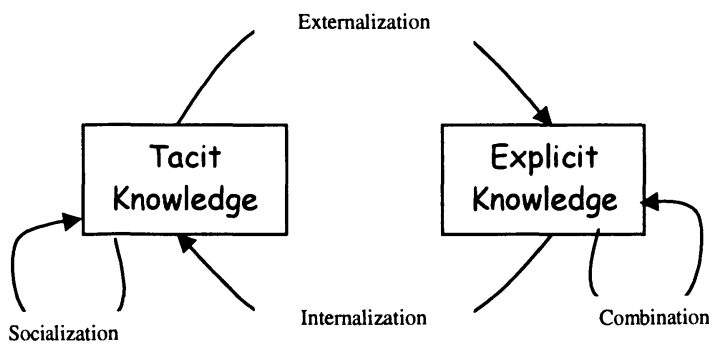

Figure 2.1. The Tacit-Explicit Knowledge Cycle

Anderson [5,6,7], Davenport [8], Harris[9], Lechner [10], Mahe [11], Stanoevska [12] and Thompson [13], have shown that the Nonaka models provide great benefits to design and implementation of real KMS in cooperative and social effects in complicated practical applications.

\subsection{Nickols Model of Knowledge in the Knowledge Management}

An alternative theory was proposed by Nickols[14]. A testable model for knowledge include the following:

- Explicit Knowledge

- Tacit Knowledge

- Implicit Knowledge

- Declaration Knowledge

- Procedural Knowledge

An important property of his model is to provide a testable procedure to distinguish different terms. There is an integration procedure to illustrate intrinsic meanings of terms in Figure 2.3.

The procedure can be explained as follows: When knowledge has been articulated, then it is explicit knowledge. Otherwise, another question is raised: Can it be articulated? If the answer is yes, then it is implicit knowledge. If the answer is no, then it is tacit knowledge. Facts and things (tasks and methods) have describing properties belong to declarative knowledge. All declarative knowledge is explicit. However, motor skills (mental skills) are doing things that correspond to procedural knowledge and all procedural knowledge is tacit. 
Nickols provides this testable procedure as the foundation of knowledge. Since other terms (declarative, procedural and strategy knowledge) can be merged into three essential terms (Explicit, Tacit and Implicit) [14], only these terms need to be handled for relationships among the components. If we view Nonaka's model as an automaton, there are four interactions among explicit and tacit knowledge (socialization from tacit to tacit, externalization from tacit to explicit, combination from explicit to explicit and internalization from explicit to tacit).

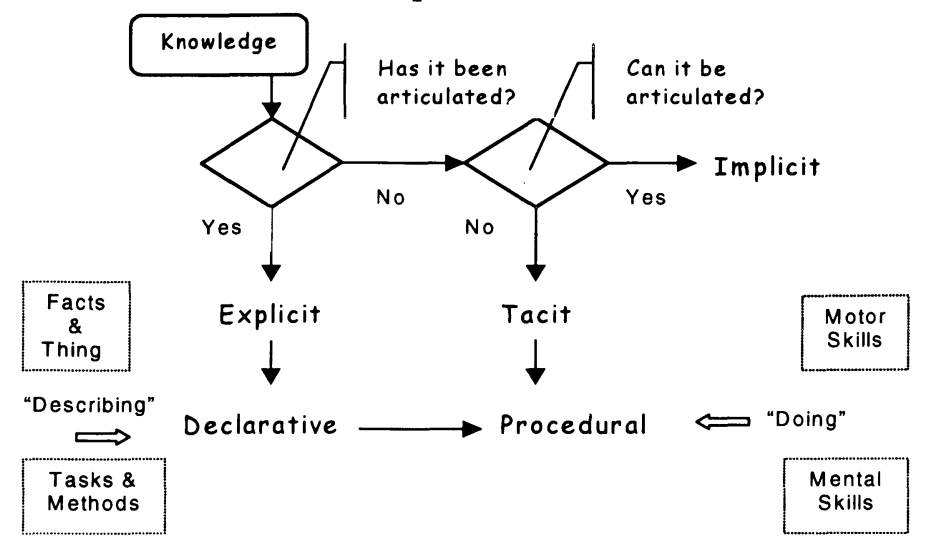

Figure 2.3. A Framework of Knowledge in Knowledge Management

Similar to Polanyi's distinctions, Nickols model is a static procedure to identify knowledge. To investigate more complex system behaviors, a dynamic model is required to integrate all three essential components of knowledge.

\section{EXECUTABLE KNOWLEDGE MODEL}

\subsection{Basic Model}

Considering three knowledge components: explicit, implicit and tacit knowledge. Explicit knowledge describes basic facts and storable document sets; tacit knowledge corresponds to personal experience, instinctive ability, spiritual knowledge and any other skills which cannot be expressed on paper or articulated. Different from above two extreme components, implicit knowledge has a bridge property that links together the explicit and tacit components. When a knowledge expert has implicit knowledge, such knowledge can be represented as explicit knowledge through knowledge discovery and description procedures. For example, a scientist developed a new idea after many years of research, the procedure is the same as to transfer tacit knowledge into implicit through externalization processes. 
When an inventor has articulated and recorded relevant knowledge as papers and description documents. Relevant implicit knowledge is transformed into explicit knowledge during procedures. Combining these considerations, we propose a new model, Executable Knowledge Model (EKM), for knowledge in knowledge management shown in Figure 3.1.

\section{Executable Knowledge Model (EKM)}

The EKM is composed of three components corresponding to three basic knowledge collections. There are four interactions among the three components under certain conditions. In addition to distinguish three basic collections, the most interesting properties of this model are its dynamic behaviors among various transformations.

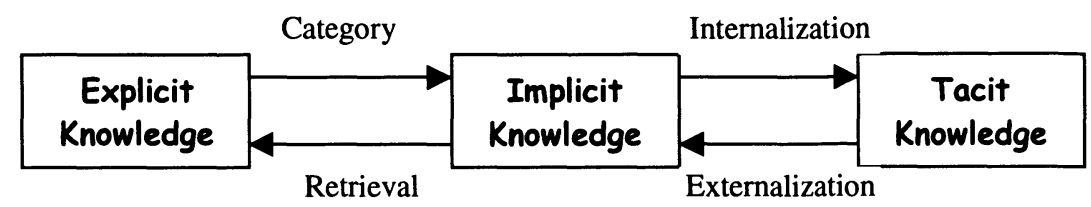

Figure 3.1. Executable Knowledge Model

\section{Category: From Explicit to Implicit}

Explicit knowledge can be manipulated and organized by various concepts and methodologies to construct higher level relationships through conceptual organization, category or classification operations. These complicated organization structures make explicit knowledge implicit. A typical example is to categorize books such as "Alice in Wonderland". Children think it is a storybook or a fairytale while mathematicians believe that it is an awesome book for training logic thought.

\section{Retrieval: From Implicit to Explicit}

From implicit knowledge, we may use content-based retrieval or creative articulation to make implicit knowledge explicit. In normal situations, there are various conditions and assumptions to add on our efforts to make such a transformation. We can imagine that a knowledge expert articulate knowledge. Complicated implicit knowledge may take much longer to be retrieved.

\section{Internalization: From Implicit to Tacit}

Internalization is a process of embodying implicit knowledge into tacit knowledge. A human expert with implicit knowledge may use internalization methodology to transform implicit knowledge to tacit. For example, a show 
pilot, amazed at the skill and poise of an Olympic diver, he tried to incorporate the diver's repertoire into his own performance. After months of studying video footage of platform divers, the pilot was inspired to perform his own twists, tumbles and pikes - all using his airplane.

\section{Externalization: From Tacit to Implicit}

Externalization is a process of presenting tacit knowledge into implicit knowledge. One example is the discovery of Benzene $\left(\mathrm{C}_{6} \mathrm{H}_{6}\right.$, the simplest aromatic hydrocarbon) structure. In 1865, German chemist August Kekule had a strange dream that six snakes were swallowing each other each head biting into another's tail. Inspired by this tacit model, he proposed the hexagonal formula with alternate single and double bonds to make this new discovery.

\subsection{Executable Model}

Using implicit knowledge as an intermediate state of knowledge, this makes possible for us to distinguish executable and non-executable collections from KMS shown in Figure 3.2.

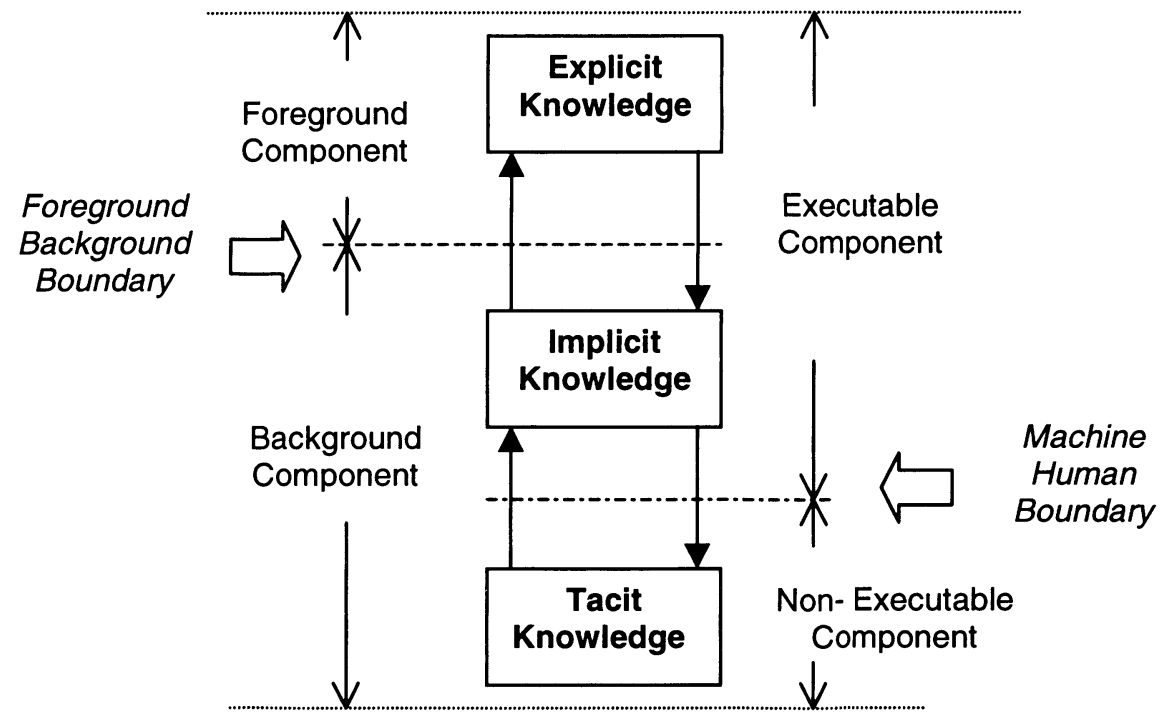

Figure 3.2 Foreground and Background, Machine and Human Knowledge Boundaries

\section{Foreground Component}

Selecting any knowledge subject belong to explicit knowledge as an object, excluding the object the rest parts of knowledge in the system correspond to its background. In general, a foreground object depends on the user's viewpoint. From a systematic consideration, foreground and 
background is corresponding to two complementary partitions. They are relative and complement each other. The relationship of these pair relations can change in different consideration under various circumstances.

However, foreground subjects can only be selected from explicit knowledge for any KMS. We cannot make a knowledge subject as foreground, if there is no way to explicitly express it. Because the reason, it is more convenient to describe the entirely explicit knowledge as foreground in KMS. Foreground component is the collection of all explicit knowledge in the KMS.

\section{Background Component}

Implicit and tacit subjects are unable to function as foreground objects in KMS. Under this consideration, we can define two collections of implicit and tacit knowledge as background component. Any knowledge subject in the implicit and tacit will be intrinsically corresponding to background. The interface between explicit and implicit knowledge is the boundary distinguishing background and foreground components in the system. This provides a natural organization to apply intrinsic knowledge properties distinguishing foreground and background components in KMS.

\section{Executable Component}

The EKM can be partitioned by another criteria. Explicit knowledge collection is composed of real materials that can be manipulated by computer or other mechanical tools. From this viewpoint, any explicit knowledge object is an executable object. However, there are hidden links among explicit knowledge objects and implicit knowledge objects. As the meanings of implicit knowledge, it will be possible to have a procedure to make implicit knowledge explicit. Therefore, when we have a super powerful mechanism to do such transformations, we can expect that it can complete almost all transformations among implicit to explicit and explicit to implicit. Under this condition, both explicit and implicit objects will be executable. Collecting all explicit and implicit objects, they are composed of an executable component in KMS.

\section{Non-Executable Component}

There is no guarantee to have a general mechanism structure to transform tacit knowledge into implicit and vice versa. From this viewpoint, the tacit component is the non-executable component of KMS. From an engineering viewpoint, it is more important for us to focus our attentions on the executable part and let human beings to use their intelligence in order for all non-executable parts to be handled correctly. 


\section{CONCLUSION}

This paper provides basic descriptions of EKM for knowledge in knowledge management. The EKM is constructed for KMS from an engineering viewpoint. Since many existing models of knowledge in knowledge management mix the processes among human beings and machine executable processes, the compounding system properties create severe difficulties for knowledge engineers to design support environments for knowledge management. The major issue in EKM is to separate executable parts from non-executable parts in KMS. This separation makes a clear boundary to handle extremely abstracted problems in various conditions.

\section{REFERENCES}

[1] Polanyi, M. (1969). Knowing and being, The University of Chicago Press.

[2] Polanyi, M. (1997). "Tacit Knowledge," Chapter 7 in Knowledge in Organizations, Laurence Prusak, Editor. Butterworth-Heinemann: Boston.

[3] Nonaka, I. (1991). "The Knowledge Creating Company." Harvard Business Review, NovemberDecember: 96-104.

[4] Nonaka, I. (1995). The Knowledge Creating Company. Oxford University Press.

[5] Anderson, J.R. (1976). Language, Memory and Thought. Erlbaum: Hillsdale.

[6] Anderson, J.R. (1993). Rules of the Mind. Erlbaum: Hillsdale.

[7] Anderson, J.R. (1995). Cognitive Psychology and Its Implications ( $4^{\text {th }}$ Edition). W.H. Freeman and Company: New York.

[8] Davenport, T. and Prusak, L. (1998). Working Knowledge. Harvard Business School Press: Boston.

[9] Harris, D. (1996) Creating a Knowledge Centric Information Technology Environment, http://www.htcs.com/ckc.html

[10] Lechner, U. and Stanoevska, K. (1998) The NetAcademy - A New Concept for Online Publishing and Knowledge Management. ACOS 98 Workshop.

[11] Mahe, H. Rieu, C., Beauchene, D. "An Original Model to Organize Know-How in a Benchmarking Context." http://spuds.cpsc.ucalgary.ca/KAW/KAW96/mahe/maherieu.html

[12] Stanoevska, K. Hombrecher, A., Handschuh, S., Schmid, B. "Efficient Information Retrieval: Tools for Knowledge Management." http://www.netacademy.org

[13] Thompson (1997) "Corporate Memories." Byte, September 1997.

[14] Nickols, F. (2000). "Knowledge in Knowledge Management." Knowledge Management Yearbook, Butterworth-Heinemann: Boston. http://home.att.net/nickols/Knowledge_in_KM.htm

[15] Belkin, N. and Croft, W. (1992) "Information Filtering and Information Retrieval: Two Sides of the Same Coin?" CACM, Vol. 35, No. 1 